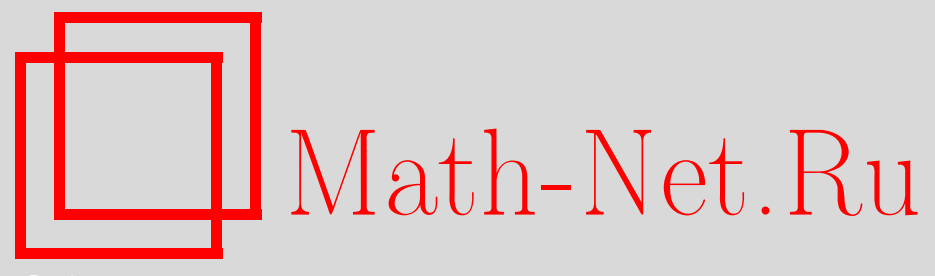

Р. В. Жалнин, В. Ф. Масягин, Е. Е. Пескова, В. Ф. Тишкин, Априорные оценки локального разрывного метода Галеркина на разнесенных сетках для решения уравнения параболического типа в рамках однородной задачи Дирихле, Вестн. Сам. гос. техн. ун-та. Сер. Физ.-мат. науки, 2020, номер 1, 116-136

DOI: https://doi.org/10.14498/vsgtu1747

Использование Общероссийского математического портала MathNet.Ru подразумевает, что вы прочитали и согласны с пользовательским соглашением

http://www.mathnet.ru/rus/agreement

Параметры загрузки:

IP : 3.80 .253 .173

26 апреля 2023 г., 10:38:39

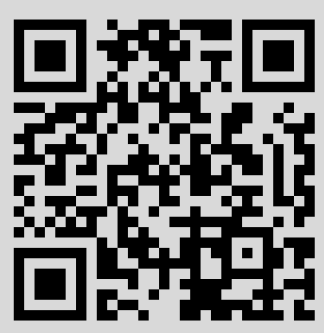


УДК 519.6

\title{
Априорные оценки локального разрывного метода Галеркина на разнесенных сетках для решения уравнения параболического типа в рамках однородной задачи Дирихле
}

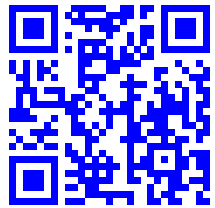

\author{
Р. В. Жалнин ${ }^{1}$, В. Ф. Масягин ${ }^{1}$, Е. Е. Пескова ${ }^{1}$, В. Ф. Тишкин \\ 1 Национальный исследовательский \\ Мордовский государственный университет имени Н. П. Огарёва, \\ Россия, 430005, Саранск, ул. Большевистская, 68. \\ 2 Институт прикладной математики им. М. В. Келдыша РАН, \\ Россия, 125047, Москва, Миусская пл., 4.
}

\section{Аннотация}

Представлены априорные оценки точности решения однородной краевой задачи для параболического уравнения с помощью локального метода Галеркина с разрывными базисными функциями на разнесенных сетках. Дискретизация по пространству строится с помощью обращения к смешанной конечно-элементной формулировке. Производные второго порядка не могут быть согласованы напрямую в слабой вариационной формулировке, используя пространство разрывных функций. Для понижения порядка компоненты вектора потока рассматриваются как вспомогательные неизвестные искомого уравнения второго порядка. Аппроксимация строится на разнесенных сетках. Основная сетка состоит из треугольников, двойственная сетка состоит из медианных контрольных объемов вокруг узлов треугольной сетки. Аппроксимация искомой функции строится на ячейках основной сетки, в то время как аппроксимация вспомогательных неизвестных строится на ячейках двойственной сетки. Для вычисления потоков на границе между элементами используется стабилизирующий параметр. При этом поток искомой функции не зависит от вспомогательных функций, в то время как поток вспомогательных величин зависит от искомой функции. Для решения поставленной задачи в работе формулируются и доказываются необходимые леммы. В результате сформулирована и доказана основная теорема, результатом которой являются априорные оценки при решении парабо-

\section{Научная статья}

(웅 Контент публикуется на условиях лицензии Creative Commons Attribution 4.0 International (https://creativecommons.org/licenses/by/4.0/deed.ru)

\section{Образец для цитирования}

Ж алнин Р. В., Масягин В. Ф., Пескова Е. Е., Тишкин В. Ф. Априорные оценки локального разрывного метода Галеркина на разнесенных сетках для решения уравнения параболического типа в рамках однородной задачи Дирихле // Becmн. Caм. гос. техн. ун-та. Сер. Физ.-мат. науки, 2020. Т. 24, № 1. С. 116-136. doi:10.14498/vsgtu1747.

\section{Сведения об авторах}

Руслан Викторович Жалнин (D) https://orcid.org/0000-0002-1103-3321

кандидат физико-математических наук, доцент; заведующий кафедрой; каф. прикладной математики, дифференциальных уравнений и теоретической механики;

e-mail: zhrv@mrsu.ru

Bиктор Федорович Масягин (1) https://orcid.org/0000-0001-6738-8183

кандидат физико-математических наук; старший научный сотрудник; каф. прикладной математики, дифференциальных уравнений и теоретической механики;

e-mail: vmasyagin@gmail.com 
лического уравнения с помощью метода Галеркина с разрывными базисными функциями. Основную роль при анализе сходимости играет оценка для отрицательной нормы градиента. В работе для стабилизирующего параметра порядка 1 показано, что порядок сходимости будет $k+1 / 2$, а в случае использования стабилизирующего параметра порядка $h^{-1}$ порядок сходимости увеличивается до $k+1$, когда в качестве базиса используются полиномы степени не выше $k$.

Ключевые слова: априорные оценки погрешности, метод конечных элементов, метод Галеркина с разрывными базисными функциями, разнесенные сетки, параболические задачи.

Получение: 4 октября 2019 г. / Исправление: 29 октября 2020 г. / Принятие: 11 ноября 2019 г. / Публикация онлайн: 16 марта 2020 г.

Введение. Ранее авторами было предложено новое семейство схем на основе локального метода Галеркина с разрывными базисными функциями на разнесенных неструктурированных сетках для уравнений диффузионного типа [1-5]. Характерной особенностью данного семейства схем является то, что аппроксимация потока искомой функции производится на двойственной сетке, состоящей из медианных контрольных объемов, связанных с узлами основной сетки, в то время как аппроксимация искомой функции рассматривается на ячейках основной сетки.

В статье представлен априорный анализ погрешности локального разрывного метода Галеркина (РМГ), или Local Discontinuous Galerkin (LDG) method, для следующей параболической задачи:

$$
\begin{array}{ll}
\frac{\partial u}{\partial t}-\Delta u=f, & \boldsymbol{x} \in \Omega, \\
u=0, & \boldsymbol{x} \in \partial \Omega, \\
\left.u\right|_{t=0}=u_{0}, & \text { в } \Omega,
\end{array}
$$

где $\Omega$ - ограниченная область в $\mathbb{R}^{d}, \partial \Omega$ - граница области $\Omega, u_{0}$ - известная функция.

Метод LDG был впервые предложен Cockburn and Shu в работе [7] как развитие численной схемы для сжимаемых уравнений Навье-Стокса, описанной Bassi and Rebay в [8]. Эта схема, в свою очередь, является развитием метода Runge-Kutta Discontinuous Galerkin (RKDG), разработанного Cockburn and $\mathrm{Shu}[9-13]$ для нелинейных гиперболических систем.

Вопросам получения априорных оценок для метода Галеркина с разрывными базисными функциями посвящено много работ как в России, так и за

\footnotetext{
Елизавета Евгенъевна Пескова (1D) https://orcid.org/0000-0003-2618-1674

кандидат физико-математических наук; младший научный сотрудник; каф. прикладной математики, дифференциальных уравнений и теоретической механики;

e-mail: e.e.peskova@mail.ru

Владимир Федорович Тишкин (1) https://orcid.org/0000-0001-7295-7002

доктор физико-математических наук, профессор, член-корреспондент РАН; заведующий отделом; e-mail:v.f.tishkin@mail.ru
} 
рубежом. Например, априорные оценки для симметричного метода Галеркина с внутренними штрафами для дискретизации по пространству эллиптических и параболических задач представлены в [17], оценки для параболических интегро-дифференциальных уравнений получены в [18], в [20] разработана абстрактная теория схем разрывного метода Галеркина в смешанной формулировке и получены априорные оценки точности для квазилинейных эллиптических уравнений второго порядка.

Наш анализ частично основывается на технике, представленной в работах $[6,16,19]$ для параболических и эллиптических задач соответственно.

Для применения локального разрывного метода Галеркина перепишем исходную параболическую задачу (1), (2) как систему уравнений в частных производных первого порядка. Введем вспомогательную переменную $\mathbf{q}=\nabla u$ и получим следующую систему уравнений:

$$
\begin{array}{ll}
\boldsymbol{q}=\nabla u, & \text { в } \Omega, \\
\frac{\partial u}{\partial t}-\nabla \cdot \boldsymbol{q}=f, & \text { в } \Omega, \\
u=0 . & \text { на } \partial \Omega, \\
\left.u\right|_{t=0}=u_{0}, & \text { в } \Omega .
\end{array}
$$

1. Локальный разрывный метод Галеркина. Покроем область расчета треугольной сеткой $\mathscr{T}_{T}$ без зазоров и наложений. Также введем в рассмотрение двойственную сетку $\mathscr{T}_{D}$, составленную из медианных ячеек, центры которых лежат в узлах ячеек треугольной сетки $\mathscr{T}_{T}$ (см. рисунок).

Для удобства дальнейших рассуждений дополнительно введем в рассмотрение сетку $\mathscr{T}_{Q}$, состоящую из ячеек $Q$, которые являются результатом пересечения ячеек из $\mathscr{T}_{T}$ и $\mathscr{T}_{D}$.

Слабое решение $(\boldsymbol{q}, u)$ системы $(3)-(5)$ будем считать определенным в пространствах $V \times \boldsymbol{W}$ :

$$
\begin{aligned}
& \boldsymbol{W}=\left\{\boldsymbol{q} \in\left(L^{2}(\Omega)\right)^{2}:\left.\boldsymbol{q}\right|_{D} \in \mathscr{H}^{1}(D)^{2}, \forall D \in \mathscr{T}_{D}\right\}, \\
& V=\left\{u \in L^{2}(\Omega):\left.u\right|_{T} \in \mathscr{H}^{1}(T), \forall T \in \mathscr{T}_{T}\right\} .
\end{aligned}
$$

Приближенное решение $\left(\boldsymbol{q}_{\boldsymbol{h}}, u_{h}\right)$ будем искать в следующих пространствах:

$$
\boldsymbol{W}_{\boldsymbol{h}}=\left\{\boldsymbol{q} \in\left(L^{2}(\Omega)\right)^{2}:\left.\boldsymbol{q}\right|_{D} \in \mathscr{P}^{k}(D)^{2}, \forall D \in \mathscr{T}_{D}\right\}
$$

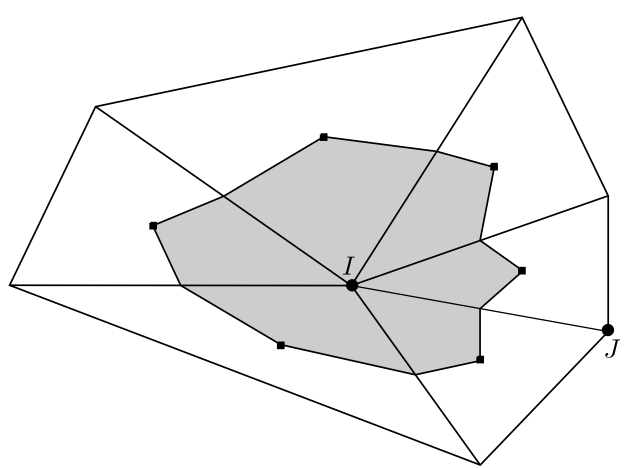

Границы ячеек основной и двойственной сетки

[The boundaries of the cells of the basic and dual mesh] 


$$
V_{h}=\left\{u \in L^{2}(\Omega):\left.u\right|_{T} \in \mathscr{P}^{k}(T), \forall T \in \mathscr{T}_{T}\right\}
$$

где $\mathscr{P}^{k}(T)$ и $\mathscr{P}^{k}(D)^{2}$ состоят из полиномов степени не выше $k$.

Для обеспечения единственности приближенного решения РМГ потребуем выполнение следующего условия:

$$
\text { если } v \in \mathscr{P}^{k}(T) \text { и } \int_{T} \nabla v \cdot \boldsymbol{w} d x=0, \forall \boldsymbol{w} \in \mathscr{P}^{k}(D)^{2}: T \cap D \neq \varnothing,
$$

то $\nabla v \equiv 0$ в $T \in \mathscr{T}_{T}$.

Приближенное слабое решение $\left(\boldsymbol{q}_{h}, u_{h}\right)$ будет определяться в каждой ячейке из следующей системы:

$$
\begin{aligned}
& \int_{D} \boldsymbol{q}_{h} \cdot \boldsymbol{w} d x+\int_{D} u_{h} \nabla \cdot \boldsymbol{w} d x-\int_{\partial D} \hat{u}_{h} \boldsymbol{w} \cdot \boldsymbol{n} d x=0 \\
& \int_{T} \frac{\partial u}{\partial t} v d x+\int_{T} \boldsymbol{q}_{h} \cdot \nabla v d x-\int_{\partial T} v \hat{\boldsymbol{q}}_{\boldsymbol{h}} \cdot \boldsymbol{n} d x=\int_{T} f v d x
\end{aligned}
$$

для всех $(\boldsymbol{w}, v) \in \mathscr{P}^{k}(D)^{2} \times \mathscr{P}^{k}(T)$, где $T \in \mathscr{T}_{T}$ и $D \in \mathscr{T}_{D}$, а численные потоки $\hat{\boldsymbol{q}}_{\boldsymbol{h}}$ и $\hat{u}_{h}$ на границе элементов зависят от значений решения с внутренней и внешней стороны ячейки.

Для определения численных потоков введем несколько обозначений. Пусть $T^{+}$и $T^{-}$- два соседних элемента триангуляции $\mathscr{T}_{T}$. Пусть $\boldsymbol{x}-$ произвольная точка грани $l=\partial T^{+} \cap \partial T^{-}$, и пусть $\boldsymbol{n}^{+}$и $\boldsymbol{n}^{-}$- соответствующие внешние нормали к элементам в данной точке. Пусть $(\boldsymbol{q}, u)$ - гладкие функции внутри каждого элемента $T^{ \pm}$и обозначим за $\left(\boldsymbol{q}^{ \pm}, u^{ \pm}\right)$следы $(\boldsymbol{q}, u)$ на $e$ из внутренности $T^{ \pm}$. После этого определим средние значения $\{[\cdot\}$ и скачки $\llbracket \cdot \rrbracket$ в точке $\boldsymbol{x} \in \boldsymbol{l}$ следующим образом:

$$
\begin{array}{ll}
\left\{\{u\}=\left(u^{+}+u^{-}\right) / 2,\right. & \left\{\{\boldsymbol{q}\}=\left(\boldsymbol{q}^{+}+\boldsymbol{q}^{-}\right) / 2,\right. \\
\llbracket u \rrbracket=u^{+} \boldsymbol{n}^{+}+u^{-} \boldsymbol{n}^{-}, & \llbracket \boldsymbol{q} \rrbracket=\boldsymbol{q}^{+} \cdot \boldsymbol{n}^{+}+\boldsymbol{q}^{-} \cdot \boldsymbol{n}^{-} .
\end{array}
$$

Если грань $l$ лежит внутри области $\Omega$, зададим потоки из $(7),(8)$ следующим образом:

$$
\begin{aligned}
& \hat{\boldsymbol{q}}=\{\{\boldsymbol{q}\}\}-C_{11} \llbracket u \rrbracket, \\
& \hat{u}=\{\{u\},
\end{aligned}
$$

где вспомогательный параметр $C_{11}$ определен в точке $\boldsymbol{x} \in \boldsymbol{l}$.

На границе потоки задаются следующим образом:

$$
\begin{aligned}
& \hat{\boldsymbol{q}}=\boldsymbol{q}^{+}-C_{11} u^{+} \boldsymbol{n}^{+} \\
& \hat{u}=0
\end{aligned}
$$


2. Используемые обозначения. Обозначим за $\Gamma_{Q}$ объединение всех ребер сетки $\mathscr{T}_{Q}$.

Просуммируем уравнения $(7),(8)$ по соответствующим элементам и получим, что приближенное решение $\left(\boldsymbol{q}_{h}, u_{h}\right)$ является единственным решением следующей вариационной задачи [15]: найти $\left(\boldsymbol{q}_{h}, u_{h}\right) \in \boldsymbol{W}_{\boldsymbol{h}} \times V_{h}$ такие, что

$$
\begin{aligned}
\int_{\Omega} \boldsymbol{q}_{h} \cdot \boldsymbol{w} d \boldsymbol{x} & +\sum_{Q \in \mathscr{T}_{Q}} \int_{Q} u_{h} \nabla \cdot \boldsymbol{w} d \boldsymbol{x}-\int_{\Gamma_{Q}}\left\{\left\{u_{h}\right\}\right\} \llbracket \boldsymbol{w} \rrbracket d s=0, \\
\left(\frac{\partial u_{h}}{\partial t}, v\right)-\sum_{Q \in \mathscr{T}_{Q}} \int_{Q} v \nabla \cdot \boldsymbol{q}_{h} d \boldsymbol{x} & +\int_{\Gamma_{Q}}\left\{\{v\} \llbracket \boldsymbol{q}_{h} \rrbracket d s+\right. \\
& +\int_{\Gamma_{Q}} C_{11} \llbracket u \rrbracket \llbracket v \rrbracket d s=\int_{\Omega} f v d \boldsymbol{x},
\end{aligned}
$$

для всех $(\boldsymbol{w}, v) \in \boldsymbol{W}_{\boldsymbol{h}} \times V_{h}$.

Введем обозначения:

$$
\begin{aligned}
& A(\boldsymbol{q}, \boldsymbol{w})=\int_{\Omega} \boldsymbol{q} \cdot \boldsymbol{w} d \boldsymbol{x} \\
& B(u, \boldsymbol{w})=\sum_{Q \in \mathscr{T}_{Q}} \int_{Q} u \nabla \cdot \boldsymbol{w} d \boldsymbol{x}-\int_{\Gamma_{Q}}\{\{u\} \llbracket \boldsymbol{w} \rrbracket d s, \\
& C(u, v)=\int_{\Gamma_{Q}} C_{11} \llbracket u \rrbracket \llbracket v \rrbracket d s, \\
& F(v)=\int_{\Omega} f v d \boldsymbol{x},
\end{aligned}
$$

где $(u, v, \boldsymbol{q}, \boldsymbol{w}) \in V_{h} \times V_{h} \times \boldsymbol{W}_{\boldsymbol{h}} \times \boldsymbol{W}_{\boldsymbol{h}}$.

С учетом введеных обозначений уравнения (11), (12) могут быть переписаны в следующем виде:

$$
\begin{aligned}
& A\left(\boldsymbol{q}-\boldsymbol{q}_{h}, \boldsymbol{w}\right)+B\left(u-u_{h}, \boldsymbol{w}\right)=0 \\
& \left(\frac{\partial u}{\partial t}-\frac{\partial u_{h}}{\partial t}, v\right)-B\left(v, \boldsymbol{q}-\boldsymbol{q}_{h}\right)+C\left(u-u_{h}, v\right)=0
\end{aligned}
$$

где $(u, \boldsymbol{q}) \in V \times \boldsymbol{W},\left(u_{h}, \boldsymbol{q}_{\boldsymbol{h}}\right) \in V_{h} \times \boldsymbol{W}_{\boldsymbol{h}}$.

Далее систему уравнений (11), (12) можно переписать в виде

$$
\left(\frac{\partial u_{h}}{\partial t}, v\right)+A\left(\boldsymbol{q}_{h}, \boldsymbol{w}\right)+B\left(u_{h}, \boldsymbol{w}\right)-B\left(v, \boldsymbol{q}_{h}\right)+C\left(u_{h}, v\right)=F(v) .
$$

3. Вспомогательные обозначения. Пусть для каждого $T \in \mathscr{T}_{T} h_{T}-$ характеристический размер $T, \rho_{T}$ - диаметр максимального шара, вложенного в $T$. Обозначим за $h=\max _{T \in \mathscr{T}_{T}} h_{T}$. Будем рассматривать триангуляцию $\mathscr{T}_{T}$, обладающую свойством регулярности, т.е. существует положительная константа $\sigma$ такая, что

$$
\frac{h_{T}}{\rho_{T}} \leqslant \sigma, \quad \forall T \in \mathscr{T}_{T} .
$$


ПреДЛОЖЕНИЕ 1. Если выполняется свойство регулярности для триангуляиии $\mathscr{T}_{T}$, то для триангуляиии $\mathscr{T}_{Q}$ такэсе выполнено свойство регулярности, т.е. существует положительная константа б такая, что

$$
\frac{h_{Q}}{\rho_{Q}} \leqslant \sigma, \quad \forall Q \in \mathscr{T}_{Q} .
$$

Доказательство. Рассмотрим произвольный треугольник $T \in \mathscr{T}_{T}$ с вершинами $T^{1}, T^{2}, T^{3}$. Обозначим середину ребра $T^{1} T^{2}$ за $T^{12}$, середину ребра $T^{1} T^{3}$ за $T^{13}$, середину ребра $T^{2} T^{3}$ за $T^{23}$, а точку пересечения меридиан за $T^{123}$. Рассмотрим четырехугольник $Q \in \mathscr{T}_{Q}$ с вершинами $T^{1}, T^{12}, T^{123}, T^{13}$ и покажем, что для него выполнено свойство регулярности.

Не умаляя общности, будем находить характеристический размер $h_{Q}$ четырехугольника $Q$ как $\sqrt{S_{Q}}$, где $S_{Q}$ - площадь $Q$. По свойству пересечения медиан получим, что $S_{Q}=\frac{1}{3} S_{T}$, где $S_{T}$ - площадь треугольника $T$.

Обозначим за $T_{1}$ треугольник с вершинами $T^{1}, T^{12}, T^{13}$. Диаметр максимального шара, вложенного в $T_{1}$, вычисляется следующим образом

$$
\rho_{T_{1}}=2 \frac{S_{T_{1}}}{p_{T_{1}}}
$$

где $p_{T_{1}}$ - полупериметр $T_{1}, S_{T_{1}}$ - площадь $T_{1}$.

Используя свойство медиан, получим

$$
\rho_{T_{1}}=\frac{1}{2} \cdot 2 \frac{S_{T}}{p_{T}}=\frac{1}{2} \rho_{T} .
$$

Далее, подставляя найденные выражения, получим

$$
\frac{h_{Q}}{\rho_{Q}}=\frac{\sqrt{S_{T}}}{\sqrt{3} \rho_{Q}} \leqslant \frac{\sqrt{S_{T}}}{\sqrt{3} \rho_{T_{1}}}=\frac{2}{\sqrt{3}} \frac{h_{T}}{\rho_{T}} \leqslant \frac{2}{\sqrt{3}} \sigma .
$$

Утверждение доказано.

Введем в рассмотрение набор $\left\langle T, T^{\prime}\right\rangle$, определенный следующим образом:

$$
\left\langle T, T^{\prime}\right\rangle=\left\{\begin{array}{cl}
\varnothing, & \text { если мера }\left(\partial T \cap \partial T^{\prime}\right)=0, \\
\text { внутренность } \partial T \cap \partial T^{\prime}, & \text { в противном случае. }
\end{array}\right.
$$

Будем предполагать, что существует положительная константа $\delta<1$ такая, что для каждого элемента $T \in \mathscr{T}_{T}$

$$
\delta \leqslant \frac{h_{T^{\prime}}}{h_{T}} \leqslant \delta^{-1} \quad \forall T^{\prime}:\left\langle T, T^{\prime}\right\rangle \neq \varnothing .
$$

Также предполагаем, что локальное пространство $\mathscr{S}(T)$ содержит пространство полиномов $\mathscr{P}^{k}(T)$ степени не выше $k$ и удовлетворяет (6).

Предполагаем, что стабилизирующий коэффициент $C_{11}$, определяющий численные потоки в (9) и (10), определяется следующим образом

$$
C_{11}(\boldsymbol{x})=\left\{\begin{array}{cl}
\zeta \min \left\{h_{T^{+}}^{\alpha}, h_{T^{-}}^{\alpha}\right\}, & \text { если } \boldsymbol{x} \in\left\langle T^{+}, T^{-}\right\rangle, \\
\zeta h_{T^{+}}^{\alpha}, & \text { если } \boldsymbol{x} \in \partial T^{+} \cap \Gamma_{\partial},
\end{array}\right.
$$


где $\zeta>0,-1 \leqslant \alpha \leqslant 0$ не зависят от размера сетки. Для удобства введем в рассмотрение параметры $\mu^{*}$ и $\mu_{*}$ :

$$
\mu^{*}=\max \{-\alpha, 1\}, \quad \mu_{*}=\min \{-\alpha, 1\} .
$$

4. Априорные оценки. Для удобства дальнейших рассуждений введем в рассмотрение проекцию. Нужно найти $\left(\tilde{u}_{h}, \tilde{\mathbf{q}}_{h}\right):[0, T] \rightarrow V_{h} \times \mathbf{W}_{h}$, удовлетворяющие соотношениям:

$$
\begin{aligned}
& A\left(\boldsymbol{q}-\tilde{\boldsymbol{q}}_{h}, \boldsymbol{w}\right)+B\left(u-\tilde{u}_{h}, \boldsymbol{w}\right)=0, \\
& -B\left(v, \boldsymbol{q}-\tilde{\boldsymbol{q}}_{h}\right)+C\left(u-\tilde{u}_{h}, v\right)=0,
\end{aligned}
$$

где $(u, \boldsymbol{q}) \in V \times \boldsymbol{W}$.

Обозначим за $\boldsymbol{\Pi}$ и $\Pi$ - проекции из $\boldsymbol{W}$ и $V$ на конечно-элементные пространства $\boldsymbol{W}_{\boldsymbol{h}}$ и $V_{\boldsymbol{h}}$ соответственно.

Используя проекции П и П, можно написать

$$
\begin{aligned}
& u-\tilde{u}_{h}=(u-\Pi u)-\left(\tilde{u}_{h}-\Pi u\right)=\eta_{u}-\xi_{u}, \\
& \boldsymbol{q}-\tilde{\boldsymbol{q}}_{h}=(\boldsymbol{q}-\Pi \boldsymbol{q})-\left(\tilde{\boldsymbol{q}}_{h}-\Pi \boldsymbol{q}\right)=\boldsymbol{\eta}_{\boldsymbol{q}}-\boldsymbol{\xi}_{\boldsymbol{q}}
\end{aligned}
$$

Используя проекцию (19), (20), систему (13), (14) можно переписать в виде

$$
\begin{aligned}
& A\left(\boldsymbol{\xi}_{\boldsymbol{q}}, \boldsymbol{w}\right)+B\left(\xi_{u}, \boldsymbol{w}\right)=0 \\
& \left(\frac{\partial \xi_{u}}{\partial t}, v\right)-B\left(v, \boldsymbol{\xi}_{\boldsymbol{q}}\right)+C\left(\xi_{u}, v\right)=\left(\frac{\partial \eta_{u}}{\partial t}, v\right)
\end{aligned}
$$

Представим ошибку погрешности проекции $(19),(20)\left(\boldsymbol{e}_{\boldsymbol{q}}, e_{u}\right)=\left(\boldsymbol{q}-\tilde{\boldsymbol{q}}_{h}, u-\tilde{u}_{h}\right)$ как следующую сумму:

$$
\left(\boldsymbol{e}_{\boldsymbol{q}}, e_{u}\right)=(\boldsymbol{q}-\boldsymbol{\Pi} \boldsymbol{q}, u-\Pi u)+\left(\boldsymbol{\Pi} \boldsymbol{e}_{\boldsymbol{q}}, \Pi e_{u}\right) .
$$

Будем считать, что выполняется свойство ортогональности метода Галеркина, а именно

$$
A\left(\boldsymbol{e}_{\boldsymbol{q}_{h}}, \boldsymbol{w}\right)+B\left(e_{u}, \boldsymbol{w}\right)-B\left(v, \boldsymbol{e}_{\boldsymbol{q}}\right)+C\left(e_{u}, v\right)=0 \quad \forall(\boldsymbol{w}, v) \in \boldsymbol{W}_{\boldsymbol{h}} \times V_{h}
$$

Для получения оценки в норме $\left\|e_{u}\right\|_{-t, D}$, где $t-$ натуральное число и $D-$ подобласть $\Omega$, нужно найти оценку погрешности аппроксимации линейного функционала $\Lambda(u)=(\lambda, u)$, где $(\cdot, \cdot)$ обозначает скалярное произведение в $L^{2}$ через $\Lambda\left(u_{h}\right)$

$$
\left\|e_{u}\right\|_{-t, D}=\sup _{\lambda \in C_{0}^{\infty}(D)} \frac{\Lambda\left(e_{u}\right)}{\|\lambda\|_{t, D}} .
$$

В настоящей работе нас интересует случай при $t=0$. Для достижения необходимых оценок введем в рассмотрение решение $\phi$ следующей двойственной задачи:

$$
-\triangle \phi=\lambda \quad \text { в } \Omega,
$$




$$
\phi=0 \quad \text { на } \partial \Omega \text {. }
$$

Приведем две леммы, содержащие всю информацию, которая будет использоваться относительно конечных элементов. Их доказательство основывается на работах $[14,15]$.

Лемма 1. Пусть $w \in H^{r+1}(T), r \geqslant 0$. Пусть П есть линейный непреръвный оператор из $H^{r+1}(T)$ в $\mathscr{S}(T)$ такой, что $\Pi w=w$ для всех $w \in \mathscr{P}^{k}(T)$. Тогда для иелого $m, 0 \leqslant m \leqslant r+1$, получим

$$
\begin{gathered}
|w-\Pi w|_{m, T} \leqslant C h_{T}^{\min \{r, k\}+1-m}\|w\|_{r+1, T}, \\
\|w-\Pi w\|_{0, \partial T} \leqslant C h_{T}^{\min \{r, k\}+\frac{1}{2}}\|w\|_{r+1, T},
\end{gathered}
$$

где $C$-константа, зависящая только от $\sigma$ в неравенстве $(16), k, d u r$.

Лемма 2. Существует положительная константа $C_{\text {inv }}$, зависящая только от $\sigma$ в неравенстве (16), $k$ и $d$ такая, что для всех $s \in \mathscr{P}^{d}(K)$ выполняется

$$
\|s\|_{0, \partial K} \leqslant C_{i n v} h_{K}^{-\frac{1}{2}}\|s\|_{0, K}
$$

для всех $K \in \mathscr{T}_{T}$ и всех $K \in \mathscr{T}_{Q}$.

Пусть П и $\boldsymbol{\Pi}$ - произвольные проекции на пространства $V_{\boldsymbol{h}}$ и $\boldsymbol{W}_{\boldsymbol{h}}$, удовлетворяющие покомпонентно предположениям леммы 1.

Лемма 3. Пусть $(\boldsymbol{q}, u) \in H^{s+1}(\Omega)^{2} \times H^{s+2}(\Omega) u(\boldsymbol{\Phi}, \phi) \in H^{t+1}(\Omega)^{2} \times$ $H^{t+2}(\Omega), s, t \geqslant 0$. Тогда справедлива следующая оченка:

$$
\begin{aligned}
\left|A(\boldsymbol{q}-\boldsymbol{\Pi} \boldsymbol{q}, \boldsymbol{w})+B\left(u_{h}, \boldsymbol{w}\right)-B(v, \boldsymbol{q}-\boldsymbol{\Pi} \boldsymbol{q})+C\left(u_{h}, v\right)\right| \leqslant \\
\leqslant C\left[\left(\sum_{Q \in \mathscr{T}_{Q}} h_{Q}^{2 \min \{s, k\}+2}\|\boldsymbol{q}\|_{s+1, Q}^{2}\right)^{1 / 2}\left(\sum_{Q \in \mathscr{T}_{Q}} h_{Q}^{2 \min \{t, k\}+2}\|\boldsymbol{\Phi}\|_{t+1, Q}^{2}\right)^{1 / 2}+\right. \\
\quad+\left(\sum_{Q \in \mathscr{T}_{Q}} h_{Q}^{2 \min \{s+1, k\}}\|u\|_{s+2, Q}^{2}\right)^{1 / 2}\left(\sum_{Q \in \mathscr{T}_{Q}} h_{Q}^{2 \min \{t, k\}+2}\|\boldsymbol{\Phi}\|_{t+1, Q}^{2}\right)^{1 / 2}+ \\
\quad+\left(\sum_{Q \in \mathscr{T}_{Q}} h_{Q}^{2 \min \{s, k\}+2}\|\boldsymbol{q}\|_{s+1, Q}^{2}\right)^{1 / 2}\left(\sum_{Q \in \mathscr{T}_{Q}} h_{Q}^{2 \min \{t+1, k\}}\|\phi\|_{t+2, Q}^{2}\right)^{1 / 2}+ \\
\left.+\left(\sum_{Q \in \mathscr{T}_{Q}} C_{11} h_{Q}^{2 \min \{s+1, k\}+1}\|u\|_{s+2, Q}^{2}\right)^{1 / 2}\left(\sum_{Q \in \mathscr{T}_{Q}} C_{11} h_{Q}^{2 \min \{t+1, k\}+1}\|\phi\|_{t+2, Q}^{2}\right)^{1 / 2}\right] .
\end{aligned}
$$

Доказательство [14]. Положим $\boldsymbol{\xi}_{\boldsymbol{q}}=\boldsymbol{q}-\boldsymbol{\Pi} \boldsymbol{q}, \xi_{u}=u-\Pi u, \boldsymbol{\xi}_{\boldsymbol{\Phi}}=$ $=\boldsymbol{\Phi}-\Pi \boldsymbol{\Phi}, \xi_{\phi}=\phi-\Pi \phi$. Тогда имеем

$$
\begin{aligned}
\mid A\left(\boldsymbol{\xi}_{\boldsymbol{q}}, \boldsymbol{\xi}_{\boldsymbol{\Phi}}\right)+ & B\left(\xi_{u}, \boldsymbol{\xi}_{\boldsymbol{\Phi}}\right)-B\left(\xi_{\phi}, \boldsymbol{\xi}_{\boldsymbol{q}}\right)+C\left(\xi_{u}, \xi_{\phi}\right) \mid \leqslant \\
& \leqslant\left|A\left(\boldsymbol{\xi}_{\boldsymbol{q}}, \boldsymbol{\xi}_{\boldsymbol{\Phi}}\right)\right|+\left|B\left(\xi_{u}, \boldsymbol{\xi}_{\boldsymbol{\Phi}}\right)\right|+\left|B\left(\xi_{\phi}, \boldsymbol{\xi}_{\boldsymbol{q}}\right)\right|+\left|C\left(\xi_{u}, \xi_{\phi}\right)\right| .
\end{aligned}
$$

Оценим отдельно каждое слагаемое. 
Из неравенства Коши-Буняковского получаем

$$
\left|A\left(\boldsymbol{\xi}_{\boldsymbol{q}}, \boldsymbol{\xi}_{\boldsymbol{\Phi}}\right)\right| \leqslant \sum_{Q \in \mathscr{T}_{Q}}\left|\int_{Q} \boldsymbol{\xi}_{\boldsymbol{q}} \boldsymbol{\xi}_{\boldsymbol{\Phi}} d x\right| \leqslant\left(\sum_{Q \in \mathscr{T}_{Q}}\left\|\boldsymbol{\xi}_{\boldsymbol{q}}\right\|_{0, Q}^{2}\right)^{1 / 2}\left(\sum_{Q \in \mathscr{T}_{Q}}\left\|\boldsymbol{\xi}_{\boldsymbol{\Phi}}\right\|_{0, Q}^{2}\right)^{1 / 2} .
$$

Далее из оценок леммы 1 следует

$$
\left|A\left(\boldsymbol{\xi}_{\boldsymbol{q}}, \boldsymbol{\xi}_{\boldsymbol{\Phi}}\right)\right| \leqslant\left(\sum_{Q \in \mathscr{T}_{Q}} h_{Q}^{2 \min \{s, k\}+2}\|\boldsymbol{q}\|_{s+1, Q}^{2}\right)^{1 / 12}\left(\sum_{Q \in \mathscr{T}_{Q}} h_{q}^{2 \min \{t, k\}+2}\|\boldsymbol{\Phi}\|_{t+1, Q}^{2}\right)^{1 / 2}
$$

Интегрируя по частям и последовательно применяя неравенство КошиБуняковского и лемму 1 , получим следующую оценку:

$$
\begin{aligned}
& \left|B\left(\xi_{u}, \boldsymbol{\xi}_{\boldsymbol{\Phi}}\right)\right|=\left|-\sum_{Q \in \mathscr{T}_{Q}}\left[\int_{Q} \nabla \xi_{u} \cdot \boldsymbol{\xi}_{\boldsymbol{\Phi}} d x+\int_{\partial Q} \boldsymbol{\xi}_{\boldsymbol{\Phi}} \cdot\left(\xi_{u} \boldsymbol{n}\right) d \sigma\right]\right| \leqslant \\
& \leqslant\left(\sum_{Q \in \mathscr{T}_{Q}}\left(\left|\xi_{u}\right|_{1, Q}^{2}+\frac{1}{h_{Q}}\left\|\xi_{u}\right\|_{0, \partial Q}^{2}\right)\right)^{1 / 2}\left(\sum_{Q \in \mathscr{T}_{Q}}\left(\left\|\boldsymbol{\xi}_{\boldsymbol{\Phi}}\right\|_{0, Q}^{2}+h_{Q}\left\|\boldsymbol{\xi}_{\boldsymbol{\Phi}}\right\|_{0, \partial Q}^{2}\right)\right)^{1 / 2} \leqslant \\
& \leqslant\left(\sum_{Q \in \mathscr{T}_{Q}} h_{Q}^{2 \min \{s+1, k\}}\|u\|_{s+2, Q}^{2}\right)^{1 / 2}\left(\sum_{Q \in \mathscr{T}_{Q}} h_{Q}^{2 \min \{t, k\}+2}\|\boldsymbol{\Phi}\|_{t+1, Q}^{2}\right)^{1 / 2} .
\end{aligned}
$$

Далее для $B\left(\xi_{\phi}, \boldsymbol{\xi}_{\boldsymbol{q}}\right)$ и $C\left(\xi_{u}, \xi_{\phi}\right)$ получим

$$
\begin{aligned}
& \left|B\left(\xi_{\phi}, \boldsymbol{\xi}_{\boldsymbol{q}}\right)\right| \leqslant\left(\sum_{Q \in \mathscr{T}_{Q}} h_{Q}^{2 \min \{s, k\}+2}\|\boldsymbol{q}\|_{s+1, Q}^{2}\right)^{1 / 2}\left(\sum_{Q \in \mathscr{T}_{Q}} h_{Q}^{2 \min \{t+1, k\}}\|\phi\|_{t+2, Q}^{2}\right)^{1 / 2} ; \\
& \left|C\left(\xi_{u}, \xi_{\phi}\right)\right|=\left|-\sum_{Q \in \mathscr{T}_{Q}} \int_{\partial Q} C_{11} \llbracket \xi_{u} \rrbracket \cdot\left(\xi_{\phi} \boldsymbol{n}\right) d \sigma\right| \leqslant \\
& \leqslant \sum_{Q \in \mathscr{T}_{Q}}\left|\int_{\partial Q} C_{11}\left(\xi_{u}^{\text {out }} \boldsymbol{n}-\xi_{u} \boldsymbol{n}\right) \cdot\left(\xi_{\phi} \boldsymbol{n}\right)\right| \leqslant 2 \sum_{Q \in \mathscr{T}_{Q}}\left|\int_{\partial Q} \sqrt{C_{11} \xi_{u}} \sqrt{C_{11}} \xi_{\phi} d \sigma\right| \leqslant \\
& \leqslant 2\left(\sum_{Q \in \mathscr{T}_{Q}} C_{11}\left\|\xi_{u}\right\|_{0, \partial Q}^{2}\right)^{1 / 2}\left(\sum_{Q \in \mathscr{T}_{Q}} C_{11}\left\|\xi_{\phi}\right\|_{0, \partial Q}^{2}\right)^{1 / 2} \leqslant \\
& \leqslant\left(\sum_{Q \in \mathscr{T}_{Q}} C_{11} h_{Q}^{2 \min \{s+1, k\}+1}\|u\|_{s+2, Q}^{2}\right)^{1 / 2}\left(\sum_{Q \in \mathscr{T}_{Q}} C_{11} h_{Q}^{2 \min \{t+1, k\}+1}\|\phi\|_{t+2, Q}^{2}\right)^{1 / 2} .
\end{aligned}
$$

Сложив полученные неравенства, получим требуемую оценку.

Следствие 1. Пусть $(\boldsymbol{q}, u) \in H^{s+1}(\Omega)^{2} \times H^{s+2}(\Omega), s \geqslant 0$ является точным решением (3)-(5), пусть $\phi \in H^{t+2}(\Omega), t \geqslant 0$ является решением двойственной задачи (24), (25) и $\boldsymbol{\Phi}=-\nabla \phi$. Полагаем такэсе, что коэббициент $C_{11}$ 
удовлетворяет выражению (18). Тогда существует константа $C$, зависящая только от $\sigma, \zeta, k$ u $d$ такая, что

$$
A(\boldsymbol{q}, \boldsymbol{\Phi})+B(u, \boldsymbol{\Phi})-B(\phi, \boldsymbol{q})+C(u, \phi) \leqslant C h^{H}\|u\|_{s+2}\|\phi\|_{t+2},
$$

где $H=1+\alpha$, когда $k=0 u H=\min \{s+1+\min \{t+1, k\}, k+1+\min \{t, k+\alpha\}\}$ для $k \geqslant 1$. Более того,

$$
A(\boldsymbol{q}, \boldsymbol{q})+B(u, \boldsymbol{q})-B(u, \boldsymbol{q})+C(u, u) \leqslant C h^{2 J}\|u\|_{s+2}\|\phi\|_{t+2},
$$

где $J=\frac{1}{2}(1+\alpha)$ при $k=0 u \quad J=\min \left\{s+1, k+\frac{1}{2}(1+\alpha)\right\}$ npu $k \geqslant 1$.

Доказательств о. Из леммы 3 получим

$$
\begin{aligned}
& \mathscr{A}(\boldsymbol{q}, u ; \boldsymbol{\Phi}, \phi) A(\boldsymbol{q}, \boldsymbol{\Phi})+B(u, \boldsymbol{\Phi})-B(\phi, \boldsymbol{q})+C(u, \phi) \leqslant \\
& \leqslant C\left[h^{\min \{s, k\}+1}\left(h^{\min \{t, k\}+1}+h^{\min \{t+1, k\}}\right)+\right. \\
&\left.+h^{\min \{s+1, k\}+1}\left(h^{\min \{t, k\}}+\zeta h^{\min \{t+1, k\}+\alpha}\right)\right]\|u\|_{s+2}\|\phi\|_{t+2}
\end{aligned}
$$

и

$$
\begin{aligned}
A(\boldsymbol{q}, \boldsymbol{q})+B(u, \boldsymbol{q})-B(u, \boldsymbol{q}) & +C(u, u) \leqslant \\
& \leqslant C\left[h^{2 \min \{s, k\}+2}+\zeta h^{2 \min \{s+1, k\}+1+\alpha}\right]\|u\|_{s+2} .
\end{aligned}
$$

ЛЕмма 4. Пусть П и П обозначают $L^{2}(\Omega)$-проекиию и $L^{2}(\Omega)^{2}$-проекиию на $V_{h}$ и $\boldsymbol{W}_{\boldsymbol{h}}$ соответственно. Тогда справедлива оченка

$$
\begin{aligned}
& A(\boldsymbol{w}, \boldsymbol{q}-\boldsymbol{\Pi} \boldsymbol{q})+B(v, \boldsymbol{q}-\boldsymbol{\Pi} \boldsymbol{q})-B(u-\Pi u, \boldsymbol{w})+C(v, u-\Pi u) \leqslant \\
& \leqslant C\left(\|\boldsymbol{w}\|_{0}^{2}+\int_{\Gamma_{Q}} C_{11} \llbracket v \rrbracket^{2} d s\right) \int_{\Gamma_{Q}}\left(\frac { 1 } { C _ { 1 1 } } \left\{\left\{\boldsymbol{\xi}_{\boldsymbol{q}}\right\}^{2}+\frac{1}{\chi}\left\{\left\{\xi_{u}\right\}^{2}+C_{11} \llbracket \xi_{u} \rrbracket^{2}\right) d s\right.\right.
\end{aligned}
$$

где $C$-константа, зависящая от $\sigma, k u d$.

Доказательств о. Возьмем $\boldsymbol{\xi}_{\boldsymbol{q}}=\boldsymbol{q}-\boldsymbol{\Pi} \boldsymbol{q}$ и $\xi_{u}=u-\Pi u$, тогда получим

$$
\begin{aligned}
\mid A\left(\boldsymbol{w}, \boldsymbol{\xi}_{\boldsymbol{q}}\right)+B\left(v, \boldsymbol{\xi}_{\boldsymbol{q}}\right)- & B\left(\xi_{u}, \boldsymbol{w}\right)+C\left(v, \xi_{u}\right) \mid \leqslant \\
& \leqslant\left|A\left(\boldsymbol{w}, \boldsymbol{\xi}_{\boldsymbol{q}}\right)\right|+\left|B\left(v, \boldsymbol{\xi}_{\boldsymbol{q}}\right)\right|+\left|B\left(\xi_{u}, \boldsymbol{w}\right)\right|+\left|C\left(v, \xi_{u}\right)\right|
\end{aligned}
$$

Используя неравенство Коши-Буняковского и тот факт, что П есть $L^{2}(\Omega)^{2}$ проекция, получим

$$
\left|A\left(\boldsymbol{w}, \boldsymbol{\xi}_{\boldsymbol{q}}\right)\right|=0
$$

Далее получим

$$
\left|B\left(v, \boldsymbol{\xi}_{\boldsymbol{q}}\right)\right|=\mid \int_{\Gamma_{Q}}\left\{\left\{\boldsymbol{\xi}_{\boldsymbol{q}}\right\} \llbracket v v \rrbracket d s \mid .\right.
$$


Умножим и поделим полученное на $C_{11}^{1 / 2}$, применим неравенство Коши-Буняковского:

$$
\left|B\left(v, \boldsymbol{\xi}_{\boldsymbol{q}}\right)\right| \leqslant\left(\int_{\Gamma_{Q}} C_{11} \llbracket v \rrbracket^{2} d s\right)^{1 / 2}\left(\int_{\Gamma_{Q}} \frac{1}{C_{11}}\left\{\left\{\boldsymbol{\xi}_{\boldsymbol{q}}\right\}^{2} d s\right)^{1 / 2} .\right.
$$

Аналогично

$$
\left|B\left(\xi_{u}, \boldsymbol{w}\right)\right|=\mid \int_{\Gamma_{Q}}\left\{\left\{\xi_{u}\right\} \llbracket \boldsymbol{w} \rrbracket d s \mid \leqslant\left(\int_{\Gamma_{Q}} \chi \llbracket \boldsymbol{w} \rrbracket^{2} d s\right)^{1 / 2}\left(\int_{\Gamma_{Q}} \frac{1}{\chi}\left\{\left\{\xi_{u}\right\}^{2} d s\right)^{1 / 2} .\right.\right.
$$

Первый множитель можно оценить с использованием леммы 2:

$$
\begin{array}{r}
\int_{\Gamma_{Q}} \chi \llbracket \boldsymbol{w} \rrbracket d s \leqslant \sum_{Q \in \mathscr{T}_{Q}} \sum_{e \in \partial Q} \int_{e} \chi\left(\left.\boldsymbol{w}\right|_{Q} \cdot \boldsymbol{n}\right)^{2} d s \leqslant \sum_{Q \in \mathscr{T}_{Q}} \chi^{\partial Q}\left\|\left.\boldsymbol{w}\right|_{Q} \cdot \boldsymbol{n}\right\|_{0, \partial Q}^{2} \leqslant \\
\leqslant C_{\text {inv }} \sup _{Q \in \mathscr{T}_{q}} \frac{\chi^{\partial Q}}{h_{Q}}\|\boldsymbol{w}\|_{0}^{2} \leqslant C_{i n v}\|\boldsymbol{w}\|_{0}^{2},
\end{array}
$$

где $\chi(\boldsymbol{x})=\min \left\{h_{Q}, h_{Q^{\prime}}\right\}$, если $\boldsymbol{x} \in\left\langle Q, Q^{\prime}\right\rangle, \chi(\boldsymbol{x})=h_{Q}$, если $\boldsymbol{x} \in \Gamma_{\partial}, \chi^{\partial Q}=$ $=\sup \{\chi(\boldsymbol{x}): \boldsymbol{x} \in \partial Q\}$.

И, наконец,

$$
\left|C\left(v, \xi_{u}\right)\right|=\left|\int_{\Gamma_{Q}} C_{11} \llbracket v \rrbracket \llbracket \xi_{u} \rrbracket d s\right| \leqslant\left(\int_{\Gamma_{Q}} C_{11} \llbracket v \rrbracket^{2} d s\right)^{1 / 2}\left(\int_{\Gamma_{Q}} C_{11} \llbracket \xi_{u} \rrbracket^{2} d s\right)^{1 / 2} .
$$

Доказательство завершено.

Лемма 5. Для $(\boldsymbol{q}, u) \in H^{s+1}(\Omega)^{2} \times H^{s+2}(\Omega), s \geqslant 0$ справедлива оиенка

$$
\begin{aligned}
\int_{\Gamma_{Q}}\left(\frac { 1 } { C _ { 1 1 } } \left\{\left\{\boldsymbol{\xi}_{\boldsymbol{q}}\right\}^{2}+\right.\right. & \left.\frac{1}{\chi}\left\{\left\{\xi_{u}\right\}\right\}^{2}+C_{11} \llbracket \xi_{u} \rrbracket^{2}\right) d s \leqslant \\
\leqslant C \sum_{Q \in \mathscr{T}_{Q}}\left(h_{Q}^{2 \min \{s, k\}+1} \frac{1}{\left.\tilde{C}_{11}^{\partial Q}\|\boldsymbol{q}\|_{s+1, Q}^{2}\right)+}\right. & +C \sum_{Q \in \mathscr{T}_{Q}}\left(h_{Q}^{2 \min \{s+1, k\}+1}\left(\tilde{C}_{11}^{\partial Q}+\frac{1}{\tilde{\chi}^{\partial Q}}\right)\|u\|_{s+2, Q}^{2}\right),
\end{aligned}
$$

где $\tilde{C}_{11}^{\partial Q}=\inf \left\{C_{11}(\boldsymbol{x}): \boldsymbol{x} \in \partial Q\right\}, \tilde{\chi}^{\partial Q}=\inf \{\chi(\boldsymbol{x}): \boldsymbol{x} \in \partial Q\}, C-$ константа, не зависящая от размера сетки, а зависящая только от аппроксимащии и констант из лемм 1 и 2, $\boldsymbol{\xi}_{\boldsymbol{q}}=\boldsymbol{q}-\boldsymbol{\Pi} \boldsymbol{q}, \xi_{u}=u-\Pi u u \chi(\boldsymbol{x})=\min \left\{h_{Q}, h_{Q^{\prime}}\right\}$, если $\boldsymbol{x} \in\left\langle Q, Q^{\prime}\right\rangle, \chi(\boldsymbol{x})=h_{Q}$, если $\boldsymbol{x} \in \Gamma_{\partial}, \chi^{\partial Q}=\sup \{\chi(\boldsymbol{x}): \boldsymbol{x} \in \partial Q\}$.

Следствие 2. Пусть $(\boldsymbol{q}, u) \in H^{s+1}(\Omega)^{2} \times H^{s+2}(\Omega), s \geqslant 0$. Полагаем, что коэффиииент $C_{11}$ удовлетворяет (18). Рассматриваемые триангуляиии удовлетворяют предположению (16). Если $\alpha \neq 0$, предполагаем, что (17) 
имеет силу. Тогда существует константа $C$, которая зависит только от $\sigma, \delta, \zeta, k$ u d такая, что

$$
\int_{\Gamma_{Q}}\left(\frac{1}{C_{11}}\left\{\left\{\boldsymbol{\xi}_{\boldsymbol{q}}\right\}^{2}+\frac{1}{\chi}\left\{\left\{\xi_{u}\right\}\right\}^{2}+C_{11} \llbracket \xi_{u} \rrbracket^{2}\right) d s \leqslant C h^{2 P}\|u\|_{s+2}^{2},\right.
$$

где $P=\frac{1}{2}\left(1-\mu^{*}\right)$, если $k=0$ и $P=\min \left\{s+\frac{1}{2}\left(1+\mu_{*}\right), k+\frac{1}{2}\left(1-\mu^{*}\right)\right\}$, если $k \geqslant 1$. Если $\alpha=0$, константа $C$ не зависит от $\delta$.

Доказ ат ель ст в о. Если взять коэффициент $C_{11}$ в виде (18), то после простых вычислений получим

$$
\frac{1}{\tilde{C}_{11}^{\partial Q}} \leqslant \zeta^{-1} h_{Q}^{-\alpha} \delta^{\alpha}
$$

и

$$
\left(C_{11}^{\partial Q}+\frac{1}{\tilde{\chi}^{\partial Q}}\right) \leqslant \zeta h_{Q}^{\alpha}+h_{Q}^{-1} \delta^{-1}
$$

где параметр $\delta$ определяется в (17).

Далее получим

$$
\begin{aligned}
\int_{\Gamma_{Q}}\left(\frac { 1 } { C _ { 1 1 } } \left\{\left\{\boldsymbol{\xi}_{\boldsymbol{q}}\right\}^{2}+\frac{1}{\chi}\left\{\left\{\xi_{u}\right\}^{2}+C_{11} \llbracket \xi_{u} \rrbracket^{2}\right) d s \leqslant\right.\right. \\
\leqslant C\left[h^{2 \min \{s, k\}+1} \zeta^{-1} h^{-\alpha}+h^{2 \min \{s+1, k\}+1}\left(\zeta h^{\alpha}+h^{-1}\right)\right]\|u\|_{s+2}^{2},
\end{aligned}
$$

откуда непосредственно вытекает искомая оценка.

Предполагаем, что выполняются следующие аппроксимационные свойства для проекций П и П:

$$
\begin{array}{r}
\mid A(\boldsymbol{q}-\boldsymbol{\Pi} \boldsymbol{q}, \boldsymbol{\Phi}-\boldsymbol{\Pi \Phi})+B(u-\Pi u, \boldsymbol{\Phi}-\boldsymbol{\Pi \Phi})-B(\phi-\Pi \phi, \boldsymbol{q}-\boldsymbol{\Pi} \boldsymbol{q})+ \\
+C(u-\Pi u, \phi-\Pi \phi) \mid \leqslant C h^{H}\|u\|_{s+2}\|\phi\|_{t+2}
\end{array}
$$

для произвольных $(\boldsymbol{q}, u),(\boldsymbol{\Phi}, \phi) \in \boldsymbol{W} \times V$ и

$$
\begin{aligned}
\mid A(\boldsymbol{w}, \boldsymbol{q}-\boldsymbol{\Pi} \boldsymbol{q}) & +B(v, \boldsymbol{q}-\boldsymbol{\Pi} \boldsymbol{q})-B(u-\Pi u, \boldsymbol{w})+ \\
& +C(v, u-\Pi u) \mid \leqslant C\left(\|\boldsymbol{w}\|_{0}^{2}+\int_{\Gamma_{Q}} C_{11} \llbracket v \rrbracket^{2} d s\right) h^{P}\|u\|_{s+2}
\end{aligned}
$$

для произвольных $(\boldsymbol{w}, v) \in \boldsymbol{W}_{\boldsymbol{h}} \times V_{h}$ и $(\boldsymbol{q}, u) \in H^{1}(\Omega)^{2} \times H^{2}(\Omega)$.

Лемма 6. Справедлива следующая оценка

$$
\left\|\boldsymbol{e}_{\boldsymbol{q}}\right\|_{0}^{2}+\int_{\Gamma_{Q}} C_{11} \llbracket e_{u} \rrbracket^{2} d s \leqslant C h^{J}\|u\|_{s+2}+C h^{P}\|u\|_{s+2} .
$$


Доказательство.

$$
\begin{aligned}
\left\|\boldsymbol{e}_{\boldsymbol{q}}\right\|_{0}^{2}+\int_{\Gamma_{Q}} C_{11} \llbracket e_{u} \rrbracket^{2} d s & \leqslant\|\boldsymbol{q}-\boldsymbol{\Pi} \boldsymbol{q}\|_{0}^{2}+ \\
& +\int_{\Gamma_{Q}} C_{11} \llbracket u-\Pi u \rrbracket^{2} d s+\left\|\boldsymbol{\Pi} \boldsymbol{e}_{\boldsymbol{q}}\right\|_{0}^{2}+\int_{\Gamma_{Q}} C_{11} \llbracket \Pi e_{u} \rrbracket^{2} d s .
\end{aligned}
$$

Т.к.

$$
\begin{gathered}
\left(\left\|\boldsymbol{\Pi} \boldsymbol{e}_{\boldsymbol{q}}\right\|_{0}^{2}+\int_{\Gamma_{Q}} C_{11} \llbracket \Pi e_{u} \rrbracket^{2} d s\right)^{2}=A\left(\boldsymbol{\Pi} \boldsymbol{e}_{\boldsymbol{q}}, \boldsymbol{\Pi} \boldsymbol{e}_{\boldsymbol{q}}\right)+B\left(\Pi e_{u}, \Pi \boldsymbol{e}_{\boldsymbol{q}}\right)- \\
-B\left(\Pi e_{u}, \boldsymbol{\Pi} \boldsymbol{e}_{\boldsymbol{q}}\right)+C\left(\Pi e_{u}, \Pi e_{u}\right), \\
=A\left(\boldsymbol{\Pi} \boldsymbol{q}-\boldsymbol{q}, \boldsymbol{\Pi} \boldsymbol{e}_{\boldsymbol{q}}\right)+B\left(\Pi u-u, \boldsymbol{\Pi} \boldsymbol{e}_{\boldsymbol{q}}\right)- \\
\quad-B\left(\Pi e_{u}, \boldsymbol{\Pi} \boldsymbol{q}-\boldsymbol{q}\right)+C\left(\Pi u-u, \Pi e_{u}\right) \text { из }(23), \\
=A\left(-\boldsymbol{\Pi} \boldsymbol{e}_{\boldsymbol{q}}, \boldsymbol{q}-\boldsymbol{\Pi} \boldsymbol{q}\right)+B\left(\Pi e_{u}, \boldsymbol{q}-\boldsymbol{\Pi} \boldsymbol{q}\right)- \\
\quad-B\left(\Pi u-u,-\boldsymbol{\Pi} \boldsymbol{e}_{\boldsymbol{q}}\right)+C\left(\Pi e_{u}, \Pi u-u\right), \\
\leqslant C\left(\left\|\boldsymbol{\Pi} \boldsymbol{e}_{\boldsymbol{q}}\right\|_{0}^{2}+\int_{\Gamma_{Q}} C_{11} \llbracket \Pi e_{u} \rrbracket^{2} d s\right) h^{P}\|u\|_{s+2} \text { из }(27) .
\end{gathered}
$$

Таким образом, справедлива следующая оценка:

$$
\left\|\Pi \boldsymbol{e}_{\boldsymbol{q}}\right\|_{0}^{2}+\int_{\Gamma_{Q}} C_{11} \llbracket \Pi e_{u} \rrbracket^{2} d s \leqslant C h^{P}\|u\|_{s+2}
$$

и далее

$$
\left\|\boldsymbol{e}_{\boldsymbol{q}}\right\|_{0}^{2}+\int_{\Gamma_{Q}} C_{11} \llbracket e_{u} \rrbracket^{2} d s \leqslant\|\boldsymbol{q}-\boldsymbol{\Pi} \boldsymbol{q}\|+\int_{\Gamma_{Q}} C_{11} \llbracket u-\Pi u \rrbracket^{2} d s+C h^{P}\|u\|_{s+2} .
$$

Искомая оценка следует после применения предположения (26).

Лемма 7. Пусть $t$-натуральное число. Тогда справедлива следующал оченка:

$$
\left\|e_{u}\right\|_{-t, D} \leqslant C h^{\min \{H, 2 P\}}\|u\|_{s+2}
$$

Доказательство. Пусть $\phi$ является решением двойственной задачи $(24),(25)$ и $\boldsymbol{\Phi}=-\nabla \phi$, тогда легко показать, что при выборе $\boldsymbol{\Phi}=-\nabla \phi$ получим

$$
A(-\boldsymbol{\Phi},-\boldsymbol{s})+B(\phi,-s)-B(w,-\mathbf{\Phi})+C(\phi, w)=\Lambda(w)
$$

для всех $(\boldsymbol{s}, w) \in \boldsymbol{W} \times V$. Задача (1), (2) может быть переписана в виде (15). Возьмем $(\boldsymbol{s}, w)=\left(\boldsymbol{e}_{\boldsymbol{q}}, e_{u}\right)$, получим

$$
\Lambda\left(e_{u}\right)=A\left(\boldsymbol{e}_{\boldsymbol{q}}, \boldsymbol{\Phi}\right)+B\left(e_{u}, \boldsymbol{\Phi}\right)-B\left(\phi, \boldsymbol{e}_{\boldsymbol{q}}\right)+C\left(e_{u}, \phi\right),
$$




$$
\begin{array}{r}
=A\left(\boldsymbol{e}_{\boldsymbol{q}}, \boldsymbol{\Phi}-\boldsymbol{\Pi \Phi}\right)+B\left(e_{u}, \boldsymbol{\Phi}-\boldsymbol{\Pi \Phi}\right)-B\left(\phi-\Pi \phi, \boldsymbol{e}_{\boldsymbol{q}}\right)+ \\
+C\left(e_{u}, \phi-\Pi \phi\right) \text { из }(23), \\
=A\left(\boldsymbol{\Pi} \boldsymbol{e}_{\boldsymbol{q}}, \boldsymbol{\Phi}-\boldsymbol{\Pi \Phi}\right)+B\left(\Pi e_{u}, \boldsymbol{\Phi}-\boldsymbol{\Pi \Phi}\right)-B\left(\phi-\Pi \phi, \boldsymbol{\Pi} \boldsymbol{e}_{\boldsymbol{q}}\right)+ \\
+C\left(\Pi e_{u}, \phi-\Pi \phi\right)+A(\boldsymbol{q}-\boldsymbol{\Pi} \boldsymbol{q}, \boldsymbol{\Phi}-\boldsymbol{\Pi} \boldsymbol{\Phi})+ \\
+B(u-\Pi u, \boldsymbol{\Phi}-\boldsymbol{\Pi \Phi})-B(\phi-\Pi \phi, \boldsymbol{q}-\boldsymbol{\Pi} \boldsymbol{q})+ \\
+C(u-\Pi u, \phi-\Pi \phi) .
\end{array}
$$

Т.к. $\left(\boldsymbol{\Pi} \boldsymbol{e}_{\boldsymbol{q}}, \Pi e_{u}\right) \in \boldsymbol{W}_{\boldsymbol{h}} \times V_{h}$, применяя (27) и (28), получим следующую оценку:

$$
\begin{aligned}
\mid A\left(\boldsymbol{\Pi} \boldsymbol{e}_{\boldsymbol{q}}, \boldsymbol{\Phi}-\boldsymbol{\Pi \Phi}\right)+B\left(\Pi e_{u}, \boldsymbol{\Phi}-\boldsymbol{\Pi \Phi}\right)-B\left(\phi-\Pi \phi, \Pi \boldsymbol{e}_{\boldsymbol{q}}\right)+ \\
+C\left(\Pi e_{u}, \phi-\Pi \phi\right) \mid \leqslant C h^{P}\|u\|_{s+2} h^{P}\|\phi\|_{s+2}
\end{aligned}
$$

далее получим

$$
\begin{aligned}
& \left|\Lambda\left(e_{u}\right)\right| \leqslant C h^{P}\|u\|_{s+2} h^{P}\|\phi\|_{s+2}+A(\boldsymbol{q}-\boldsymbol{\Pi} \boldsymbol{q}, \boldsymbol{\Phi}-\boldsymbol{\Pi \Phi})+ \\
& \quad+B(u-\Pi u, \boldsymbol{\Phi}-\boldsymbol{\Pi \Phi})-B(\phi-\Pi \phi, \boldsymbol{q}-\boldsymbol{\Pi} \boldsymbol{q})+C(u-\Pi u, \phi-\Pi \phi) .
\end{aligned}
$$

Применим предположение (26) и по определению негативной нормы получим искомую оценку.

СледСтвиЕ 3. Пусть $t$ - натуральное число. Тогда справедлива следующая оценка:

$$
\left\|e_{u t}\right\|_{-t, D} \leqslant C h^{\min \{H, 2 P\}}\left(\|u\|_{s+2}+\left\|u_{t}\right\|_{s+2}\right)
$$

До каз а m eль ст во. Для получения искомой оценки нужно продифференцировать (19), (20) по $t$ и выполнить процедуру доказательства, аналогичную при доказательстве леммы 7.

Используя введенную ранее проекцию (19), (20), можно записать

$$
\begin{aligned}
& u-u_{h}=\left(u-\tilde{u}_{h}\right)-\left(u_{h}-\tilde{u}_{h}\right)=\eta_{u}-\xi_{u} \\
& \mathbf{q}-\boldsymbol{q}_{h}=\left(\mathbf{q}-\tilde{\boldsymbol{q}}_{h}\right)-\left(\boldsymbol{q}_{h}-\tilde{\boldsymbol{q}}_{h}\right)=\boldsymbol{\eta}_{\boldsymbol{q}}-\boldsymbol{\xi}_{\boldsymbol{q}}
\end{aligned}
$$

Лемма 8. Существует константа $C$, не зависящая от $h$ и р такая, что

$$
\left\|\xi_{u}\right\|^{2}+\int_{0}^{t}\left\|\boldsymbol{\xi}_{\boldsymbol{q}}\right\|^{2} d s \leqslant C\left(\left\|\xi_{u}(0)\right\|^{2}+\int_{0}^{T}\left\|\eta_{u_{t}}\right\|^{2} d s\right) .
$$

Доказательств о. Возьмем $\boldsymbol{w}=\boldsymbol{\xi}_{\boldsymbol{q}}$ в $(21)$ и $v_{h}=\xi_{u}$ в $(22)$, просуммируем и получим

$$
\frac{1}{2} \frac{d}{d t}\left\|\xi_{u}\right\|^{2}+A\left(\boldsymbol{\xi}_{\boldsymbol{q}}, \boldsymbol{\xi}_{\boldsymbol{q}}\right)+C\left(\xi_{u}, \xi_{u}\right)=\left(\eta_{u_{t}}, \xi_{u}\right) .
$$


Используя неравенство Коши-Буняковского, получим

$$
\frac{d}{d t}\left\|\xi_{u}\right\|^{2}+\left\|\boldsymbol{\xi}_{\boldsymbol{q}}\right\|^{2}+2 C\left(\xi_{u}, \xi_{u}\right) \leqslant\left\|\eta_{u_{t}}\right\|^{2}+\left\|\xi_{u}\right\|^{2}
$$

Интегрируя последнее выражение от 0 до $t$, получим

$$
\left\|\xi_{u}\right\|^{2}+\int_{0}^{t}\left\{\left\|\boldsymbol{\xi}_{\boldsymbol{q}}\right\|^{2}+2 C\left(\xi_{u}, \xi_{u}\right)\right\} d s \leqslant\left\|\xi_{u}(0)\right\|^{2}+\int_{0}^{t}\left\|\eta_{u_{t}}\right\|^{2} d s+\int_{0}^{t}\left\|\xi_{u}\right\|^{2} d s .
$$

Используя лемму Гронуолла, получим

$$
\left\|\xi_{u}\right\|^{2}+\int_{0}^{t}\left\|\boldsymbol{\xi}_{\boldsymbol{q}}\right\|^{2} d s \leqslant C\left(\left\|\xi_{u}(0)\right\|^{2}+\int_{0}^{T}\left\|\eta_{u_{t}}\right\|^{2} d s\right)
$$

Возьмем $\boldsymbol{w}_{\boldsymbol{h}}=\boldsymbol{\xi}_{\boldsymbol{q}_{\boldsymbol{t}} \text { в }}(21)$ и $v_{\boldsymbol{h}}=\xi_{u_{t}}$ в $(22)$. Используя неравенство КошиБуняковского, получим

$$
\frac{1}{2} \frac{d}{d t} A\left(\boldsymbol{\xi}_{\boldsymbol{q}}, \boldsymbol{\xi}_{\boldsymbol{q}}\right)+\frac{1}{2}\left\|\xi_{u_{t}}\right\|^{2}+\frac{1}{2} \frac{d}{d t} C\left(\xi_{u}, \xi_{u}\right) \leqslant \frac{1}{2}\left\|\eta_{u_{t}}\right\|^{2}
$$

Интегрируя от 0 до $t$, получим

$$
\left\|\boldsymbol{\xi}_{\boldsymbol{q}}\right\|^{2}+\int_{0}^{t}\left\|\xi_{u_{t}}\right\|^{2} d s+J\left(\xi_{u}, \xi_{u}\right) \leqslant\left\|\boldsymbol{\xi}_{\boldsymbol{q}}(0)\right\|^{2}+C\left(\xi_{u}(0), \xi_{u}(0)\right)+\int_{0}^{t}\left\|\eta_{u_{t}}\right\|^{2} d s
$$

\section{5. Формулировка и доказательство основной теоремы.}

Теорема. Пусть $(\boldsymbol{q}, u)$ является решением задачи (3)-(5) и $\left(\boldsymbol{q}_{h}, u_{h}\right)$ является решением задачи (11)-(12). Пусть выполнены предположсения на локальные пространства и на вид стабилизирующего параметра $C_{11}$. Предполагаем, что триангуляиия $\mathscr{T}_{T}$ удовлетворяет предположению (16). При $\alpha \neq 0$ также предполагаем, что имеет силу предположение (17). Тогда для $(\boldsymbol{q}, u) \in H^{s+1}(\Omega) \times H^{s+2}(\Omega)$ при $s \geqslant 0$ получим оценку

$$
\left\|u-u_{h}\right\|_{0} \leqslant C h^{P+D}\left(\|u\|_{s+2}+\int_{0}^{t}\left\{\|u(s)\|_{s+2}+\left\|u_{s}(s)\right\|_{s+2}\right\} d s\right),
$$

где $C$ зависит от $\sigma, \delta$ (в случае $\alpha \neq 0), \zeta, k u d$;

$$
P=\min \left\{s+\frac{1}{2}\left(1+\mu_{*}\right), k+\frac{1}{2}\left(1-\mu^{*}\right)\right\}, \quad D=\frac{1}{2}\left(1+\mu_{*}\right), \quad \text { если } \quad k \geqslant 1 .
$$

$B$ случае $k=0$ получим $P=D=\frac{1}{2}\left(1-\mu_{*}\right)$.

Доказательство. С учетом неравенств треугольника для искомой оценки справедливо утверждение

$$
\left\|u-u_{h}\right\|_{0} \leqslant\left\|u-\tilde{u}_{h}\right\|_{0}+\left\|u_{h}-\tilde{u}_{h}\right\|_{0} .
$$

Рассмотрим норму $L^{2}$ погрешности $u-u_{h}$. Возьмем $t=0$ и $D=\Omega$ в лемме 7. Из условия эллиптической регулярности двойственной задачи (24), (25) 
получим $\|\phi\|_{2} \leqslant C\|\lambda\|_{0}$. Оценка $\left\|u-\tilde{u}_{h}\right\|_{0}$ получается из следствий 1 и 2 и ограниченности $\|\Phi\|_{1}$ и $\|\phi\|_{2}$ величиной $\|\lambda\|_{0}$. Получим

$$
\left\|u-\tilde{u}_{h}\right\|_{0} \leqslant C h^{\min \left\{\left.H\right|_{t=0}, P+\left.P\right|_{s=0}\right\}}\|u\|_{s+2},
$$

и т.к. $\min \left\{\left.H\right|_{t=0}, P+\left.P\right|_{s=0}\right\}=P+\left.P\right|_{s=0}$, получим следующую оценку

$$
\left\|u-\tilde{u}_{h}\right\|_{0} \leqslant C h^{P+D}\|u\|_{s+2},
$$

где $D=\left.P\right|_{s=0}$. Используя неравенство треугольника $(29)$, лемму 8 и следствие 3, получим искомую оценку. Доказательство завершено.

В таблице ниже представлены порядки сходимости по $h$ с различным выбором стабилизирующего параметра $C_{11}$. Эти порядки получаются из (30).

Порядки сходимости решения $u \in H^{s+2}$ для $s \geqslant 0$ и $k \geqslant 1$

[Convergence orders of solution $u \in H^{s+2}$ for $s \geqslant 0$ and $k \geqslant 1$ ]

\begin{tabular}{|c|c|c|}
\hline & $C_{11}$ & $\left\|u-u_{h}\right\|_{0}$ \\
\hline$\alpha=0$ & $O(1)$ & $\min \left\{s+\frac{1}{2}, k\right\}+\frac{1}{2}$ \\
$\alpha=-1$ & $O(1 / h)$ & $\min \{s+1, k\}+1$ \\
\hline
\end{tabular}

Заключение. В работе получены оценки погрешности решения двумерной однородной краевой задачи для параболического уравнения с помощью метода Галеркина с разрывными базисными функциями на разнесенных неструктурированных сетках. При этом предполагалось, что узлы двойственной сетки являются центрами ячеек основной сетки. Как видно из таблицы, в случае использования стабилизирующего коэффициента порядка единицы получается порядок сходимости $k+1 / 2$, а в случае использования стабилизирующего коэффициента порядка $h^{-1}$ порядок сходимости увеличивается до $k+1$ для исследуемого метода, где $k$ - максимальный порядок используемых полиномов в базисных функциях. В данном случае, в отличие от традиционного подхода, в котором используется одна сетка, выбор численных потоков на границе элементов происходит интуитивно более понятно за счет использования разнесенных сеток. Оптимальность полученных теоретических результатов тестировалась для двумерных задач в серии ранее опубликованных работ $[1,2,4,5]$. Полученные порядки сходимости для локального разрывного метода Галеркина на разнесенных неструктурированных сетках соответствуют аналогичным оценкам, ранее полученным другими авторами на неразнесенных неструктурированных сетках $[7,15,18]$.

Конкурирующие интересы. Конкурирующих интересов не имеем.

Авторский вклад и ответственность. Все авторы принимали участие в разработке концепции статьи и в написании рукописи. Авторы несут полную ответственность за предоставление окончательной рукописи в печать. Окончательная версия рукописи была одобрена всеми авторами.

Финансирование. Работа выполнена при поддержке РФФИ (проекты 18-41-130001, 18-31-00102), Минобрнауки РФ (1.6958.2017/8.9) и гранта Президента РФ для молодых российских ученых - кандидатов наук (MK-2007.2018.1). Работа В. Ф. Тишкина выполнена при поддержке гранта РНФ (проект 17-71-30014). 


\section{Библиографический список}

1. Масягин В. Ф., Жалнин Р. В., Тишкин В. Ф. О применении разрывного конечноэлементного метода Галеркина для решения двумерных уравнений диффузионного типа на неструктурированных сетках // Журнал CBMO, 2013. Т. 15, № 2. С. 59-65.

2. Жалнин Р. В., Ладонкина М. Е., Масягин В. Ф., Тишкин В. Ф. Об одном способе решения уравнений диффузионного типа с помощью разрывного метода Галёркина на неструктурированной сетке // Журнал СВМО, 2014. Т. 16, № 2. С. 7-13.

3. Жалнин Р. В., Ладонкина М. Е., Масягин В. Ф., Тишкин В. Ф. Решение трехмерных уравнений теплопроводности с помощью разрывного метода Галёркина на неструктурированных сетках // Вестн. Сам. гос. техн. ун-та. Сер. Физ.-мат. науки, 2015. Т. 19, № 3. C. 523-533. doi : 10.14498/vsgtu1351.

4. Жалнин Р. В., Ладонкина М. Е., Масягин В. Ф., Тишкин В. Ф. Решение задач о нестационарной фильтрации вещества с помощью разрывного метода Галеркина на неструктурированных сетках // Ж. вычисл. матем. и матем. физ., 2016. Т. 56, №6. С. 989-998. doi : $10.7868 / \mathrm{S} 0044466916060247$.

5. Жалнин Р. В., Ладонкина М. Е., Масягин В. Ф., Тишкин В. Ф. Применение разрывного метода Галеркина для решения параболических задач в анизотропных средах на треугольных сетках // Вестн. ЮУрГУ. Сер. Матем. моделирование и программирование, 2016. T. 9, № 3. C. 144-151. doi : 10.14529/mmp160313.

6. Жалнин Р. В., Масягин В. Ф. Априорные оценки для метода Галеркина с разрывными базисными функциями на разнесенных сетках для однородной задачи Дирихле // Вестн. ЮУрГУ. Сер. Матем. моделирование и программирование, 2018. Т. 11, № 2 . C. 29-43. doi: 10.14529/mmp180203.

7. Cockburn B., Shu C.-W. The local discontinuous Galerkin finite element method for convection-diffusion systems // SIAM J. Numer. Anal., 1998. vol. 35, no. 6. pp. 2440-2463. doi : 10.1137/S0036142997316712.

8. Bassi F., Rebay S. A High-Order Accurate Discontinuous Finite Element Method for the Numerical Solution of the Compressible Navier-Stokes Equations // J. Comp. Phys., 1997. vol. 131, no. 2. pp. 267-279. doi : 10.1006/jcph.1996.5572.

9. Cockburn B., Hou S., Shu C.-W. TVB Runge-Kutta local projection discontinuous Galerkin finite element method for conservation laws IV: The multidimensional case // Math. Comp., 1990. vol. 54, no. 190. pp. 545-581. doi : 10.1090/S0025-5718-1990-1010597-0.

10. Cockburn B., Lin S.-Y., Shu C.-W. TVB Runge-Kutta local projection discontinuous Galerkin finite element method for conservation laws III: One dimensional systems // J. Comput. Phys., 1989. vol.84, no.1. pp. 90-113. doi : 10.1016/0021-9991(89)90183-6.

11. Cockburn B., Shu C.-W. TVB Runge-Kutta local projection discontinuous Galerkin finite element method for conservation laws. II. General framework // Math. Comp., 1989. vol. 52, no. 186. pp. 411-435. doi: 10.1090/S0025-5718-1989-0983311-4.

12. Cockburn B., Lin S.-Y., Shu C.-W. The Runge-Kutta local projection $P^{1}$-discontinuous Galerkin method for scalar conservation laws // ESAIM: Mathematical Modelling and Numerical Analysis, 1991. vol. 25, no.3. pp. 337-361. doi: 10.1051/m2an/1991250303371.

13. Cockburn B., Shu C.-W. The Runge-Kutta discontinuous Galerkin finite element method for conservation laws V: Multidimensional systems // J. Comput. Phys., 1998. vol. 141, no. 2. pp. 199-224. doi: 10.1006/jcph.1998.5892.

14. Ciarlet P. G. The Finite Element Method for Elliptic Problems / Classics in Applied Mathematics. Philadelphia: SIAM, 2002. xxiii+529 pp. doi: 10.1137/1.9780898719208.

15. Castillo P., Cockburn B., Perugia I., Schötzau D. An a priory error analysis of the local discontinuous Galerkin method for elliptic problems // SIAM J. Numer. Anal., 2000. vol. 38, no. 5. pp. 1676-1706. doi : 10.1137/S0036142900371003.

16. Thomée V. Galerkin Finite Element Methods for Parabolic Problems/ Springer Series in Computational Mathematics. vol. 25. Berlin: Springer, 1997. $\mathrm{x}+302$ pp. doi: 10.1007/ 978-3-662-03359-3. 
17. Rivière B. Discontinuous Galerkin Methods for Solving Elliptic and Parabolic Equations/ Frontiers in Applied Mathematics. Philadelphia: SIAM, 2008. xxii+178 pp. doi: 10.1137/ 1.9780898717440.

18. Pany A., Yadav S. An $h p$-local discontinuous Galerkin method for parabolic integrodifferential equations // J. Sci. Comput., 2011. vol.46, no.1. pp. 71-99. doi:10.1007/ s10915-010-9384-z.

19. Babuška I., Suri M. The $h-p$ version of the finite element method with quasiuniform meshes // ESAIM: Mathematical Modelling and Numerical Analysis, 1987. vol.21, no. 2. pp. 199-238. doi: 10.1051/m2an/1987210201991.

20. Даутов Р. З., Федотов Е. М. Абстрактная теория HDG-схем для квазилинейных эллиптических уравнений второго порядка // Ж. вычисл. матем. и матем. физ., 2014. T. 54, № 3. C. 463-480. doi : 10.7868/S0044466914030041. 
MSC: $65 \mathrm{N30}$

\title{
A priori error estimates of the local discontinuous Galerkin method on staggered grids for solving a parabolic equation for the homogeneous Dirichlet problem
}

\author{
R. V. Zhalnin ${ }^{1}$, V. F. Masyagin ${ }^{1}$, E. E. Peskova ${ }^{1}$, V. F. Tishkin ${ }^{2}$ \\ 1 Ogarev Mordovia State University, \\ 68, Bol'shevistskaya st., Saransk, 430005, Russian Federation. \\ 2 Keldysh Institute of Applied Mathematics of Russian Academy of Sciences, \\ 4, Miusskaya pl., Moscow, 125047, Russian Federation.
}

\begin{abstract}
In this paper, we present a priori error analysis of the solution of a homogeneous boundary value problem for a second-order differential equation by the Discontinuous Galerkin method on staggered grids. The spatial discretization is constructed using an appeal to a mixed finite element formulation. Second-order derivatives cannot be directly matched in a weak variational formulation using the space of discontinuous functions. For lower the order, the components of the flow vector are considered as auxiliary variables of the desired second-order equation. The approximation is based on staggered grids. The main grid consists of triangles, the dual grid consists of median control volumes around the nodes of the triangular grid. The approximation of the desired function is built on the cells of the main grid, while the approximation of auxiliary variables is built on the cells of the dual grid. To calculate the flows at the boundary between the elements, a stabilizing parameter is used. Moreover, the flow of the desired function does not depend on auxiliary functions, while the flow of auxiliary variables depends on the desired function. To solve this problem, the necessary lemmas are formulated and proved. As a result, the main theorem is formulated and proved, the result of which is a priori estimates for solving a parabolic equation using the discontinuous Galerkin method. The main role in the analysis of convergence is played by the estimate for the negative norm of the gradient. We show that for stabilization parameter of first order, the $L^{2}$-norm of the solution is of order $k+1 / 2$, if stabilization parameter of order $h^{-1}$
\end{abstract}

\section{Research Article}

๖ (ㅇ)( The content is published under the terms of the Creative Commons Attribution 4.0 International License (http://creativecommons.org/licenses/by/4.0/)

Please cite this article in press as:

Zhalnin R. V., Masyagin V. F., Peskova E. E., Tishkin V. F. A priori error estimates of the local discontinuous Galerkin method on staggered grids for solving a parabolic equation for the homogeneous Dirichlet problem, Vestn. Samar. Gos. Tekhn. Univ., Ser. Fiz.-Mat. Nauki [J. Samara State Tech. Univ., Ser. Phys. Math. Sci.], 2020, vol. 24, no. 1, pp. 116-136. doi: 10.14498/vsgtu1747 (In Russian).

\section{Authors' Details:}

Ruslan V. Zhalnin (1) https://orcid.org/0000-0002-1103-3321

Cand. Phys. \& Math. Sci., Associate Professor; Dept. Head; Dept. of Applied Mathematics, Differential Equations and Theoretical Mechanics; e-mail: zhrv@mrsu.ru

Victor F. Masyagin (1) https://orcid.org/0000-0001-6738-8183

Cand. Phys. \& Math. Sci.; Senior Researcher; Dept. of Applied Mathematics, Differential Equations and Theoretical Mechanics; e-mail: vmasyagin@gmail.com 
is taken, the order of convergence of the solution increases to $k+1$, when polynomials of total degree at least $k$ are used.

Keywords: a priori error analysis, finite element method, discontinuous Galerkin method, staggered grids, parabolic problems.

Received: $4^{\text {th }}$ October, 2019 / Revised: $29^{\text {th }}$ October, $2020 /$

Accepted: $11^{\text {th }}$ November, $2019 /$ First online: $16^{\text {th }}$ March, 2020

Competing interests. We have no competing interests.

Authors' contributions and responsibilities. Each author has participated in the article concept development and in the manuscript writing. The authors are absolutely responsible for submitting the final manuscript in print. Each author has approved the final version of manuscript.

Funding. This work was supported by the Russian Foundation for Basic Research Research (project nos. 18-41-130001, 18-31-00102), the Ministry of Education and Science of the Russian Federation (1.6958.2017/8.9), and the Programme of the President of the Russian Federation for the support of young scientists (grant no. MK-2007.2018.1). The work of Vladimir F. Tishkin was supported by the grant from the Russian Science Foundation (grant no. 17-71-30014).

\section{References}

1. Masyagin V. F., Zhalnin R. V., Tishkin V. F. Discontinuous finite-element Galerkin method for numerical solution of two-dimensional diffusion problems on unstructured grids, Zhurnal SVMO, 2013, vol. 15, no. 2, pp. 59-65 (In Russian).

2. Zhalnin R. V., Ladonkina M. E., Masyagin V. F., Tishkin V. F. Discontinuous finite-element Galerkin method for numerical solution of two-dimensional diffusion problems on unstructured grids, Zhurnal SVMO, 2014, vol.16, no. 2, pp. 7-13 (In Russian).

3. Zhalnin R. V., Ladonkina M. E., Masyagin V. F., Tishkin V. F. Solution of 3D heat conduction equations using the discontinuous Galerkin method on unstructured grids, Vestn. Samar. Gos. Tekhn. Univ., Ser. Fiz.-Mat. Nauki [J. Samara State Tech. Univ., Ser. Phys. Math. Sci.], 2015, vol. 19, no. 3, pp. 523-533 (In Russian). doi : 10.14498/vsgtu1351.

4. Zhalnin R. V., Ladonkina M. E., Masyagin V. F., Tishkin V. F. Solving the problem of non-stationary filtration of substance by the discontinuous Galerkin method on unstructured grids, Comput. Math. Math. Phys., 2016, vol.56, no.6, pp. 977-986. doi: 10.1134/ S0965542516060245.

5. Zhalnin R. V., Ladonkina M. E., Masyagin V. F., Tishkin V. F. Discontinuous finite-element Galerkin method for numerical solution of parabolic problems in anisotropic media on triangle grids, Bulletin of the South Ural State University. Series: Mathematical Modelling, Programming and Computer Software, 2016, vol.9, no. 3, pp. 144-151 (In Russian). doi : $10.14529 / \mathrm{mmp} 160313$.

6. Zhalnin R. V., Masyagin V. F. Galerkin method with discontinuous basis functions on staggered grips a priory estimates for the homogeneous Dirichlet problem, Bulletin of the South Ural State University. Series: Mathematical Modelling, Programming and Computer Software, 2018, vol.11, no. 2, pp. 29-43 (In Russian). doi: 10.14529/mmp180203.

Elizaveta E. Peskova (D) https://orcid.org/0000-0003-2618-1674

Cand. Phys. \& Math. Sci.; Junior Researcher; Dept. of Applied Mathematics, Differential Equations and Theoretical Mechanics; e-mail: e.e.peskova@mail.ru

Vladimir F. Tishkin (D) https://orcid.org/0000-0001-7295-7002

Dr. Phys. \& Math. Sci., Professor; Corresponding Member of Russian Academy of Sciences; Dept. Head; e-mail: v.f.tishkin@mail.ru 
7. Cockburn B., Shu C.-W. The local discontinuous Galerkin finite element method for convection-diffusion systems, SIAM J. Numer. Anal., 1998, vol. 35, no. 6, pp. 2440-2463. doi : 10.1137/S0036142997316712.

8. Bassi F., Rebay S. A High-Order Accurate Discontinuous Finite Element Method for the Numerical Solution of the Compressible Navier-Stokes Equations, J. Comp. Phys., 1997, vol. 131, no. 2, pp. 267-279. doi : 10.1006/jcph.1996.5572.

9. Cockburn B., Hou S., Shu C.-W. TVB Runge-Kutta local projection discontinuous Galerkin finite element method for conservation laws IV: The multidimensional case, Math. Comp., 1990, vol. 54, no. 190, pp. 545-581. doi: 10.1090/S0025-5718-1990-1010597-0.

10. Cockburn B., Lin S.-Y., Shu C.-W. TVB Runge-Kutta local projection discontinuous Galerkin finite element method for conservation laws III: One dimensional systems, J. Comput. Phys., 1989, vol. 84, no. 1, pp. 90-113. doi: 10.1016/0021-9991(89)90183-6.

11. Cockburn B., Shu C.-W. TVB Runge-Kutta local projection discontinuous Galerkin finite element method for conservation laws. II. General framework, Math. Comp., 1989, vol. 52, no. 186, pp. 411-435. doi : 10.1090/S0025-5718-1989-0983311-4.

12. Cockburn B., Lin S.-Y., Shu C.-W. The Runge-Kutta local projection $P^{1}$-discontinuous Galerkin method for scalar conservation laws, ESAIM: Mathematical Modelling and Numerical Analysis, 1991, vol. 25, no.3, pp. 337-361. doi: 10.1051/m2an/1991250303371.

13. Cockburn B., Shu C.-W. The Runge-Kutta discontinuous Galerkin finite element method for conservation laws V: Multidimensional systems, J. Comput. Phys., 1998, vol. 141, no. 2, pp. 199-224. doi: 10.1006/jcph.1998.5892.

14. Ciarlet P. G. The Finite Element Method for Elliptic Problems, Classics in Applied Mathematics. Philadelphia, SIAM, 2002, xxiii+529 pp. doi: 10.1137/1.9780898719208.

15. Castillo P., Cockburn B., Perugia I., Schötzau D. An a priory error analysis of the local discontinuous Galerkin method for elliptic problems, SIAM J. Numer. Anal., 2000, vol. 38, no. 5, pp. 1676-1706. doi : 10.1137/S0036142900371003.

16. Thomée V. Galerkin Finite Element Methods for Parabolic Problems, Springer Series in Computational Mathematics, vol.25. Berlin, Springer, 1997, x+302 pp. doi: 10.1007/ 978-3-662-03359-3.

17. Rivière B. Discontinuous Galerkin Methods for Solving Elliptic and Parabolic Equations, Frontiers in Applied Mathematics. Philadelphia, SIAM, 2008, xxii+178 pp. doi: 10.1137/ 1.9780898717440 .

18. Pany A., Yadav S. An $h p$-local discontinuous Galerkin method for parabolic integrodifferential equations, J. Sci. Comput., 2011, vol.46, no.1, pp. 71-99. doi:10.1007/ s10915-010-9384-z.

19. Babuška I., Suri M. The $h-p$ version of the finite element method with quasiuniform meshes, ESAIM: Mathematical Modelling and Numerical Analysis, 1987, vol.21, no. 2, pp. 199-238. doi: 10.1051/m2an/1987210201991.

20. Dautov R. Z., Fedotov E. M. Abstract theory of hybridizable discontinuous Galerkin methods for second-order quasilinear elliptic problems, Comput. Math. Math. Phys., 2014, vol. 54, no. 3, pp. 474-490. doi: 10.1134/S096554251403004X. 